\title{
A tutorial on the selection and installation of surge protection devices in a TT wiring system
}

\begin{abstract}
This paper provides an easy-to-understand guidance for the selection and installation of transient protection devices, especially in a TT wiring system. Several fringe issues with respect to surge protection have also been discussed. The information presented can be used as educational material that guides electrical engineers in addressing lightning protection issues of LV power systems and ELV signal systems. A number of real life examples and survey outcomes that reflect misinterpretation of standards and scientific practices, gathered in the South Asian region have also been discussed.
\end{abstract}

